\title{
Carotenóides da cianobactéria Synechocystis pevalekii produzida em condições normais e sob limitação de nutrientes
}

\author{
Marcos Coelho Müller ${ }^{1}$, Delia B. Rodriguez-Amaya ${ }^{1 *}$, Sergio O. Lourenço ${ }^{2}$ \\ ${ }^{1}$ Departamento de Ciência de Alimentos, Faculdade de Engenharia de Alimentos, Universidade Estadual de \\ Campinas, ${ }^{2}$ Departamento de Biologia Marinha, Universidade Federal Fluminense
}

*Correspondência:

D. B. Rodriguez-Amaya

Departamento de Ciência de

Alimentos

Faculdade de Engenharia de

Alimentos, Universidade Estadual de

Campinas

E-mail: delia@fea.unicamp.br
O uso de microalgas e cianobactérias como fontes de nutrientes e substâncias bioativas para alimentos e suplementos alimentares vem despertando grande interesse nos últimos anos. Por meio de cromatografia em coluna aberta com espectrofotometria de absorção, cromatografia líquida de alta eficiência com detector de conjunto de diodos, cromatografia em camada delgada e reações de grupos funcionais, foram identificados trans- $e$ cis- $\beta$-caroteno, equininona, $\beta$-criptoxantina, 3-hidroxi-4'-cetocarotenóide, zeaxantina e 3,3-diidroxi-4'-cetocarotenóide em Synechocystis pevalekii. A cianobactéria Synechocystis pevalekii apresentou-se verde em condições normais de cultivo devido à presença de clorofilas. Com o cultivo em condições de "stress" (redução de $80 \%$ dos nutrientes do meio Conway original), as clorofilas desapareceram e a cianobactéria apresentou coloração laranja. $O \beta$-caroteno diminuiu de 307 para $248 \mu \mathrm{g} / \mathrm{g}$ e a $\beta$-criptoxantina de 94 para $13 \mu \mathrm{g} / \mathrm{g}$. Por outro lado, a zeaxantina aumentou de 29 para $220 \mu \mathrm{g} / \mathrm{g}$. S. pevalekii, portanto, apresenta potencial comercial como fonte de zeaxantina, carotenóide apontado como responsável pela ação protetora contra a degeneração macular e catarata, junto com a luteína. Os resultados demonstram que as condições de produção da cianobatéria podem ser estabelecidas de tal forma que a biossintese de carotenóides importantes para saúde humana, de difícil obtenção, seja favorecida. Já existem várias fontes comerciais de $\beta$-caroteno, mas são raras as fontes de zeaxantina.
Unitermos

- Cianobactéria

- Synechochystis pevalekii

- Carotenóides

- Zeaxantina

\section{INTRODUÇÃO}

Dentre os pigmentos naturais, os carotenóides sobressaem-se devido às suas propriedades e funções. Além de atuarem como corante, possuem funções fisiológicas como atividade pró-vitamínica A, fortalecimento do sistema imunológico e redução do risco de doenças degenerativas como câncer, doenças cardiovasculares, catarata e degeneração macular (Krinsky, 1993; Mayne, 1996; Olson, 1999). 
Embora sofram forte competição com os carotenóides sintéticos produzidos na Europa e EUA, os extratos naturais de carotenóides continuam tendo um lugar no mercado internacional. Este é o caso do urucum, do açafrão e da páprika.

Os carotenóides são pigmentos amplamente distribuídos na natureza, encontrados em todos os tecidos fotossintetizantes, fungos, bactérias, leveduras, algas, animais e também em partes não fotossintéticas de plantas, como frutas, flores, sementes e raízes.

Atualmente, microalgas e cianobactérias constituem-se em fontes para obtenção de carotenóides. As microalgas vêm sendo investigadas para uso em alimentos ou rações por mais de quatro décadas. Mais recentemente, o principal interesse é a sua utilização na produção de uma gama de compostos como polissacarídeos, lipídeos, pigmentos, vitaminas, esteróis, enzimas, antibióticos e farmacêuticos (Borowitzka, 1988; Kay, 1991). O cultivo de microalgas, como Dunaliella salina, pode ser ao ar livre, assim demandando investimento relativamente baixo na construção e manutenção do sistema de produção de biomassa.

Existem hoje fábricas bem estabelecidas para produção de microalgas e cianobactérias em Taiwan (Chlorella), Tailândia (Spirulina), EUA (Spirulina, Dunaliella, Haematococcus), Austrália (Dunaliella, Chlorella), Israel (Dunaliella), República Tcheca (Scenedesmus) e México (Spirulina) (Borowitzka, 1993; Gouveia et al., 1996). Instalações menores estão operando ou estão sendo implantadas na Índia, China, Vietnã, Chile, França, Espanha e África do Sul.

Para a ampliação da comercialização de produtos de microalgas e cianobactérias, maiores estudos são necessários. Isso inclui a caracterização química de novas espécies, bem como a investigação detalhada da biologia e fisiologia das espécies escolhidas. Para manter a competitividade, algumas providências são necessárias, como a completa utilização da biomassa, a reciclagem do meio de cultura, a produção durante o ano, o baixo risco de contaminação, o baixo custo da coleta da biomassa e da obtenção de ingredientes estáveis e eficazes (Gouveia et al., 1996).

Os carotenóides primários são sintetizados por microalgas sob condições normais, favoráveis ao crescimento celular. Em condições de "stress", as microalgas sintetizam carotenóides secundários como um mecanismo de defesa. A formação de carotenóides secundários pode ser provocada pela limitação de nitrogênio, luz de alta intensidade, $\mathrm{pH}$, salinidade e presença de outros compostos orgânicos (Ben-Amotz et al., 1982; Rise et al., 1994; Orosa et al., 2001).
O objetivo deste estudo foi determinar a composição de carotenóides de uma espécie nativa, contribuindo para o conhecimento sobre os recursos naturais brasileiros. Além disso, foi também estudado o efeito do cultivo da espécie sob limitação por nutrientes na composição de carotenóides da biomassa obtida.

\section{MATERIAL E MÉTODOS}

\section{Cultivo da Cianobactéria}

Os cultivos de Synechocystis pevalekii, isolada do litoral de Ubatuba, São Paulo, foram realizados no Instituto de Biologia da Universidade Federal do Rio de Janeiro, sob as condições descritas a seguir: aeração $(2,0 \mathrm{~L}$ de ar/min.), temperatura de $22 \pm 1{ }^{\circ} \mathrm{C}$ e em ciclos de $12 \mathrm{~h}$ de

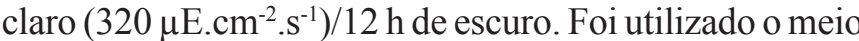
Conway (Walne, 1966), com salinidade de 32\%. Para o cultivo de $S$. pevalekii realizado sob estresse de nutrientes, os nutrientes foram reduzidos a $20 \%$ do meio Conway original. O cultivo normal e sob limitação de nutrientes foram realizados simultaneamente.

Para verificar a cinética de crescimento da cianobatéria, alíquotas foram retiradas diariamente por 18 dias. Para a caracterização dos carotenóides, realizada em duplicata, a amostragem foi feita no $15^{\circ}$ dia, quando os cultivos encontravam-se em fase estacionária de crescimento.

\section{Análise de Carotenóides por Cromatografia em Coluna Aberta}

A cromatografia em coluna aberta (CCA) foi realizada de acordo com Rodriguez-Amaya (1999), adaptando-a às características da cianobactéria.

Primeiramente, foram avaliados dois procedimentos de extração, comumente usados em laboratórios. O procedimento 1 é o que é tradicionalmente utilizado para a extração de carotenóides de microalgas e cianobactérias. O procedimento 2 é usado em nosso laboratório para verduras, frutas e outros alimentos.

Procedimento 1: A amostra foi centrifugada, para que se retirasse o sobrenadante. As células sedimentadas, contendo carotenóides e clorofilas, foram sucessivamente centrifugadas com acetona, até que toda coloração fosse retirada, isto é, até que o solvente continuasse incolor ou o sedimento esbranquiçado.

Procedimento 2: A amostra foi triturada em um almofariz, junto com acetona gelada e Hiflosupercel. Em seguida, foi feita a filtração a vácuo, utilizando um funil sinterizado (porosidade 2). Este processo foi repetido até que o solvente ficasse incolor ou o resíduo, esbranquiçado. 
Os carotenóides foram transferidos de acetona para éter de petróleo, em um funil de separação, com auxílio da água, adicionando o extrato aos poucos até que todos os carotenóides fossem transferidos para o éter de petróleo. Após três ou quatro lavagens, a solução etérea de carotenóides foi recolhida.

Para efetuar a saponificação, ao extrato de pigmentos em éter de petróleo, foi adicionada solução metanólica de $\mathrm{KOH} 10 \%$ contendo $0,1 \%$ de butil-hidroxitolueno (BHT), na proporção de 1:1, e a mistura foi deixada por cerca de $12 \mathrm{~h}$, à temperatura ambiente, no escuro. O álcali foi eliminado por sucessivas lavagens com água, até obter-se $\mathrm{pH}$ neutro. Após recolhimento da fase superior, foi adicionado sulfato de sódio anidro para retirar vestígios de água do extrato.

O extrato foi concentrado a vácuo em rotoevaporador com temperatura máxima de $35^{\circ} \mathrm{C}$, a concentração foi completada em corrente de nitrogênio.

A separação dos carotenóides foi conseguida em uma coluna de vidro de $2,5 \mathrm{~cm}$ de diâmetro e de $28,0 \mathrm{~cm}$ de altura, empacotada com mistura de $\mathrm{MgO}$ :Hiflosupercel (1:1) a altura de $12 \mathrm{~cm}$. Uma pequena quantidade de sulfato de sódio anidro foi adicionada $(2 \mathrm{~cm})$ no topo da coluna para reter eventual água residual do extrato.

Mantendo o vácuo, a coluna foi molhada com éter de petróleo. Após regulagem do fluxo, foi feita a aplicação do extrato concentrado de carotenóides. A fase móvel foi constituída de combinações de éter de petróleo, éter etílico e acetona.

Foi estabelecido o seguinte perfil de eluição: $50 \mathrm{~mL}$ cada de éter de petróleo puro e $1 \%$ de éter etílico; $100 \mathrm{~mL}$ de $2 \%$ de éter etílico; $50 \mathrm{~mL}$ de $15 \%$ de acetona; $30 \mathrm{~mL}$ de $20 \%$ de acetona; $50 \mathrm{~mL}$ cada de $25 \%, 30 \%, 40 \%, 50 \%$, $60 \%, 70 \%, 80 \%$ de acetona com todos os solventes citados em éter de petróleo e, finalmente, $50 \mathrm{~mL}$ de acetona pura e $20 \%$ de água em acetona.

No caso da Synechocystis pevalekii, cultivada em condição de estresse, a eluição foi feita da seguinte forma: $50 \mathrm{~mL}$ de éter de petróleo puro; $15 \mathrm{~mL}$ de $1 \%$ de éter etílico; $20 \mathrm{~mL}$ de $2 \%$ de éter etílico; $50 \mathrm{~mL}$ cada de $5 \%$ e $8 \%$ de éter etílico; $30 \mathrm{~mL}$ de $1 \%$ de acetona, $40 \mathrm{~mL}$ de $2 \%$ de acetona, $50 \mathrm{~mL}$ cada de $5 \%$ e $10 \%$ de acetona; $30 \mathrm{~mL}$ de $15 \%$ de acetona; $50 \mathrm{~mL}$ de $20 \%$ de acetona; $40 \mathrm{~mL}$ de $30 \%$ de acetona, $20 \mathrm{~mL}$ de $40 \%$ de acetona, $50 \mathrm{~mL}$ cada de $50 \%, 60 \%$ de acetona, $20 \mathrm{~mL}$ de $70 \%$ de acetona, $30 \mathrm{~mL}$ de $80 \%$ de acetona com todos os solventes citados acima em éter de petróleo e, finalmente, $50 \mathrm{~mL}$ de acetona e $20 \%$ de água em acetona.

As frações contendo acetona foram lavadas com água destilada em funil de separação e recolhidas. Após adição de sulfato de sódio anidro, as frações foram decan- tadas, concentradas ou diluídas a um volume adequado para leitura.

O espectro de absorção de cada fração separada por CCA foi registrado na faixa de comprimento de onda de 300 a $550 \mathrm{~nm}$, utilizando um espectrofotômetro marca Beckman, modelo DU-640. Os comprimentos de onda de absorção máxima $\left(\lambda_{\max }\right)$ foram comparados com os valores tabelados por Davies (1976) e Britton (1995).

Um conjunto de parâmetros foi utilizado para a identificação dos carotenóides: (1) o comportamento cromatográfico em CCA, cromatografia líquida de alta eficiência (CLAE) e cromatografia em camada delgada (CCD) de sílica-gel desenvolvida com 5\% de metanol em tolueno; (2) espectro de absorção; (3) reações químicas.

Na placa de sílica-gel, desenvolvida com $5 \%$ de metanol em tolueno, os carotenos eluíam junto com a frente de solvente e as xantofilas ficavam retidas em menor ou maior grau, conforme o número e tipo de grupos funcionais.

Para verificar a presença de carotenóides epoxidados, a placa desenvolvida foi exposta a vapores de $\mathrm{HCl}$ concentrado por 3 a 5 minutos. A presença de epoxicarotenóides foi manifestada pela mudança da colaboração amarela para azul.

No caso de um apocarotenol, foi necessária a reação de redução, que consistiu na colocação de cristais de boroidreto de sódio à solução do pigmento dissolvido em etanol $95 \%$ e a mistura deixada durante três horas na geladeira. A resposta positiva foi manifestada pela transformação da única máxima de absorção, característica de cetocarotenóide, em três máximas, típicas de hidroxicarotenóides e abaixamento do $\mathrm{R}_{\mathrm{F}}$ (fator de retenção) na camada delgada.

Para comprovar a presença de hidroxilas, utilizou-se a reação de acetilação. Esta reação consistiu em juntar 0,2 $\mathrm{mL}$ de anidrido acético ao pigmento já dissolvido em piridina (cerca de 2,0 mL). A mistura foi deixada por 21 horas no escuro à temperatura ambiente. Após a transferência do carotenóide ao éter de petróleo, a reação positiva foi demonstrada pelo aumento do $\mathrm{R}_{\mathrm{F}}$ na camada delgada.

Havendo a confirmação da presença de hidroxila, foi necessário saber se estava em posição alílica ou não e para isso foi necessária outra reação, a metilação. Ao pigmento dissolvido em metanol $(2,0 \mathrm{~mL})$, foram adicionadas 5 gotas de $\mathrm{HCl}$ 0,2 N. Após três horas de reação, à temperatura ambiente e no escuro, o pigmento foi transferido para éter de petróleo. A reação positiva foi manifestada pelo aumento do valor de $\mathrm{R}_{\mathrm{F}}$ na camada delgada.

\section{Quantificação dos Carotenóides}

A cianobactéria ainda com meio de cultivo foi mis- 
turada por agitação manual leve. Para determinar o peso por volume, uma alíquota com volume definido foi retirada e filtrada para separar o meio da cianobactéria e o líquido extracelular na cianobactéria retirado por aquecimento a $50{ }^{\circ} \mathrm{C}$ por 4 horas (condições determinadas em testes preliminares). Para a determinação quantitativa, uma outra alíquota foi utilizada e os carotenóides extraídos, transferidos para éter de petróleo e separados cromatograficamente, como discutido anteriormente. A concentração de cada carotenóide foi determinada espectrofotometricamente de acordo com Davies (1976).

\section{Análise de Carotenóides por CLAE}

Os carotenóides foram também analisados por CLAE, utilizando um cromatógrafo líquido marca Varian, modelo 9010, com detector de conjunto de diodos marca Waters, modelo 994, e integrador marca Varian modelo 4400. A coluna empregada foi Vydac 218TP54, $\mathrm{C}_{18}, 5 \mu \mathrm{m}$, 4,6 mm x $250 \mathrm{~mm}$.

Para a amostra de S. pevalekii não saponificada, foi utilizada, como fase móvel, metanol: tetraidrofurano (99:1), de modo isocrático, com vazão de $0,8 \mathrm{~mL} / \mathrm{min}$. Para a amostra saponificada e a amostra cultivada sob "stress", a proporção foi alterada para 98:2. Cada corrida no método CLAE durou 40 minutos.

\section{RESULTADOS E DISCUSSÃO}

As curvas de crescimento de Synechocystis pevalekii cultivadas em condições normais e sob limitação de nutrientes estão apresentadas na Figura 1. Os dois tratamentos foram iniciados com uma densidade de $6 \times 10^{5}$ células/mL e após 24 horas o cultivo com meio de cultura completo passou a apresentar maior densidade de células $(p<0,01$, teste $t)$. As diferenças entre tratamentos foram se intensificando ao longo do crescimento dos cultivos, culminando com a produção de células cerca de quatro vezes maior no controle.

\section{Carotenóides de Synechocystis pevalekii}

Ambos os procedimentos para a extração de carotenóides mostraram-se eficientes. $\mathrm{O}$ procedimento 2 tem a vantagem de ser mais simples (não necessitando de uma centrífuga), econômico e rápido.

Na cianobactéria $S$. pevalekii foram identificados por CCA e espectrofotometria de absorção um caroteno e cinco xantofilas. A Figura 2 mostra a separação dos carotenóides na coluna de $\mathrm{MgO}$ :Hiflosupercel e na placa de sílica gel desenvolvida por 5\% de metanol em tolueno. As estruturas dos carotenóides identificados estão na Figura 3.

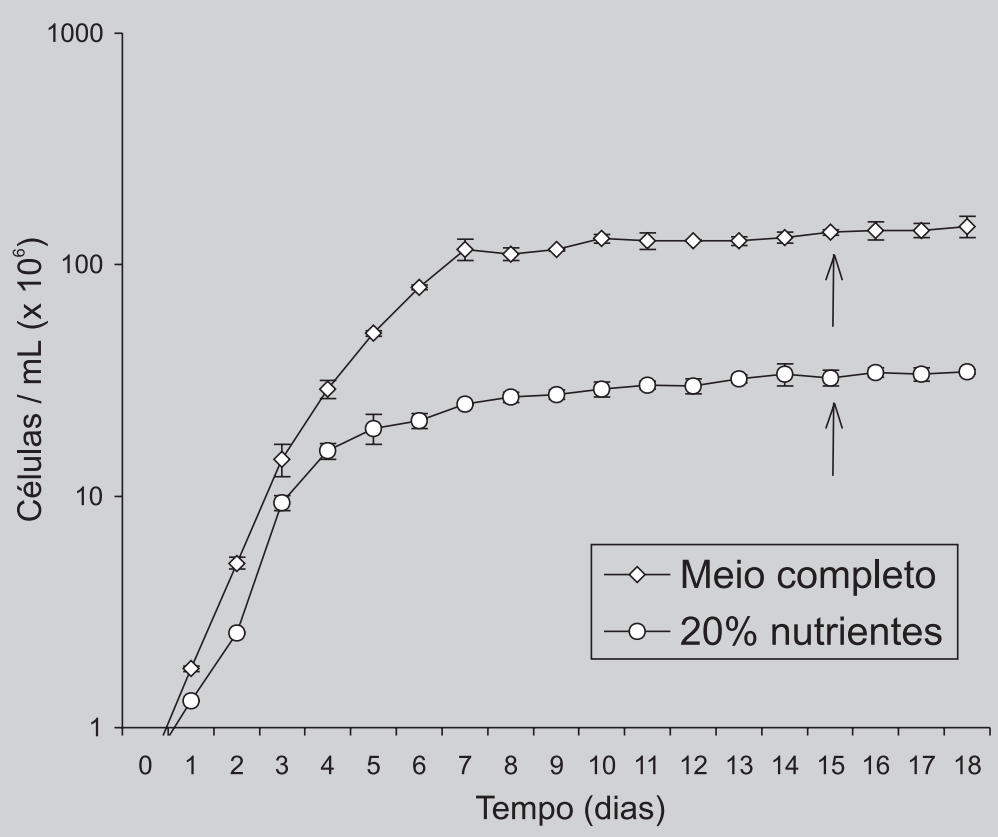

FIGURA 1 - Curvas de crescimento de Synechocystis pevalekii em condições normais e sob limitação de nutrientes. As setas indicam o momento em que as amostras foram retiradas para as análises de carotenóides. 


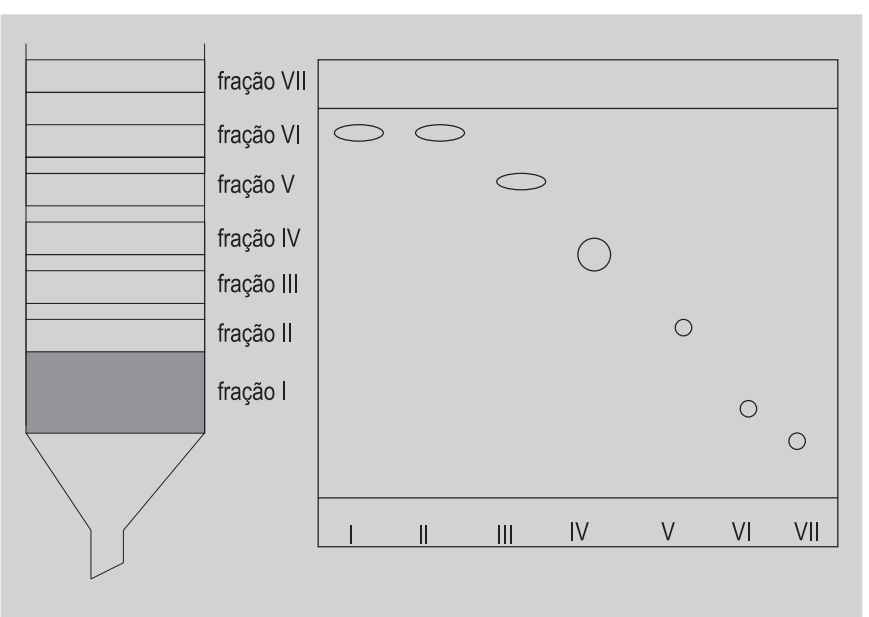

FIGURA 2 - Separação de carotenóides de S. pevalekii na coluna de MgO: Hiflosupercel (1:1) e na placa de sílica-gel desenvolvida por $5 \%$ de metanol em tolueno.

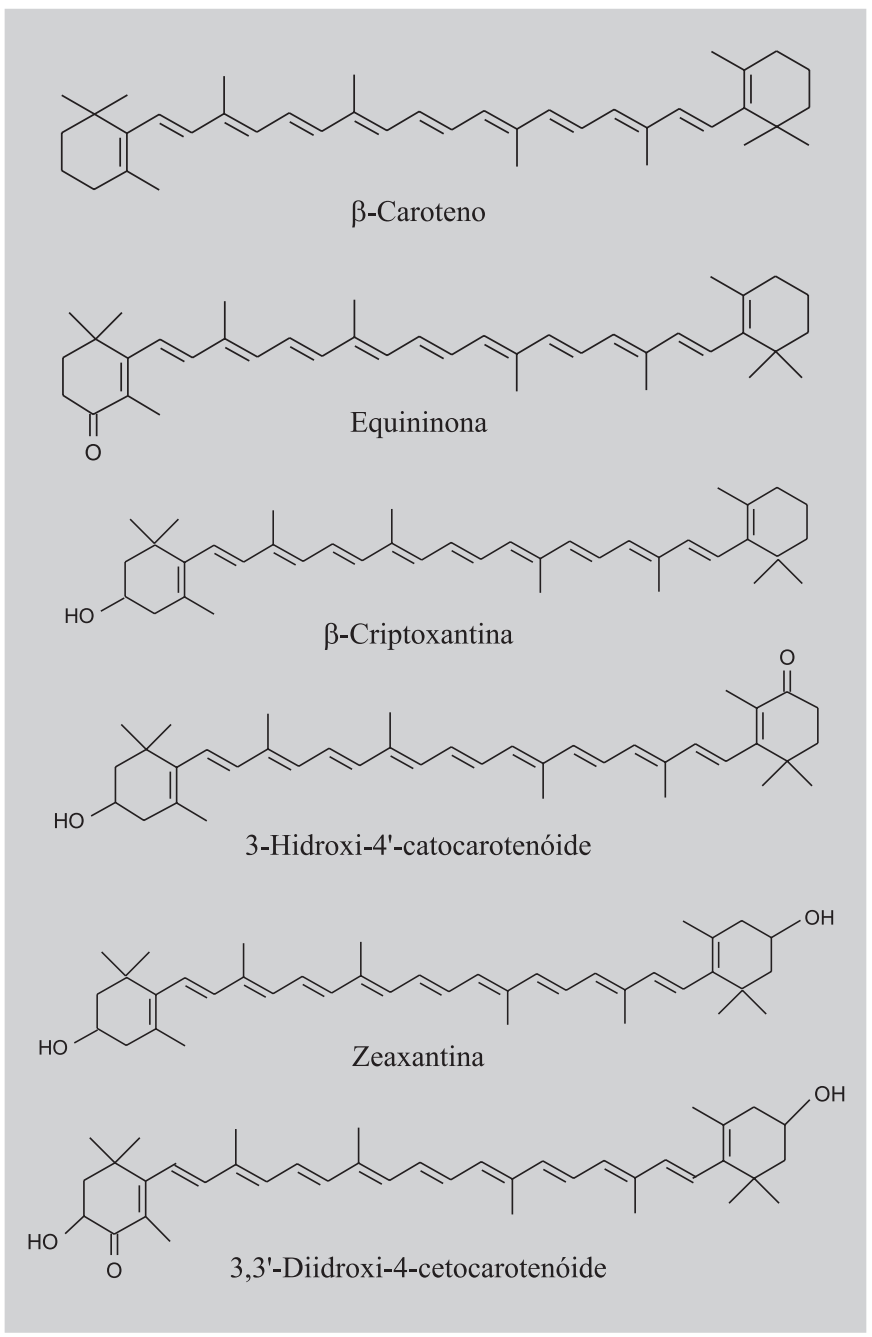

FIGURA 3 - Carotenóides identificados em Synechocystis pevalekii.
Na seguinte discussão da identificação, os $\lambda_{\text {max }}$ citados são referentes aos espectros dos carotenóides em éter de petróleo e os $\mathrm{R}_{\mathrm{F}}$ são os obtidos em placa de sílica-gel desenvolvida por $5 \%$ de metanol em tolueno.

A primeira fração foi identificada, conclusivamente, como $\beta$-caroteno ( $\beta, \beta$-caroteno) através do espectro de absorção característico e comportamento cromatográfico. Esta fração, com coloração laranja forte, foi eluída da coluna com 2-5\% de éter etílico em éter de petróleo. Seu $\mathrm{R}_{\mathrm{F}} \mathrm{de}$ 0,97 na camada de sílica-gel foi típico de um caroteno. $\mathrm{O}$ espectro de absorção com $\lambda_{\text {max }}$ a 447 e $475 \mathrm{~nm}$ e ombro a $425 \mathrm{~nm}$ refletiu a sua estrutura bicíclica com 11 duplas ligações conjugadas.

A segunda fração foi eluída com 2-8\% de acetona em éter de petróleo. Seu espectro indicou mistura, tendo $\lambda_{\text {max }} \mathrm{a}$ $376,400,424,445 \mathrm{~nm}$ e um ombro a $470 \mathrm{~nm}$. O R foi o de um caroteno. Foi feita recromatografia em outra coluna de MgO: Hyflosupercel (1:1), mas devido à baixa quantidade, não foi possível a sua purificação e identificação.

A terceira fração foi eluída com 5-10\% de acetona em éter de petróleo e apresentou coloração rosa. Seu espectro foi característico de um cetocarotenóide, consistindo de um pico largo com $\lambda_{\text {max }}$ a $452 \mathrm{~nm}$. Após a reação de redução, o espectro se transformou em três picos com $\lambda_{\text {max }}$ a 447 e $472 \mathrm{~nm}$ e um ombro a $423 \mathrm{~nm}$, refletindo a formação de hidroxila a partir de um grupo carbonílico. A presença deste grupo hidroxílico foi confirmada pela reação positiva a acetilação e sua posição alílica pela resposta positiva à metilação. Todas estas características identificaram, conclusivamente, a fração III como equininona $(\beta, \beta$-caroten-4-ona).

A quarta fração, apresentando coloração alaranjada, foi eluída da coluna com $10-20 \%$ de acetona em éter de petróleo. Seu espectro revelou cromóforo semelhante ao de $\beta$-caroteno com $\lambda_{\text {max }}$ a 446 e $473 \mathrm{~nm}$ e um ombro a $425 \mathrm{~nm}$. O Rf de 0,52 foi típico de um carotenóide monoidroxilado. Foram realizadas as reações de metilação e acetilação, tendo resultados negativo e positivo, respectivamente, e o produto acetilado apresentando um $\mathrm{R}_{\mathrm{F}}$ de 0,90 . Assim, a hidroxila não estava em posição alílica e o carotenóide foi confirmado como $\beta$-criptoxantina ( $\beta$, $\beta$-caroten-3-ol).

A quinta fração (coloração rósea) foi eluída da coluna com $15-30 \%$ de acetona em éter de petróleo. Seu espectro foi característico de um cetocarotenóide, tendo pico largo com $\lambda_{\text {max }}$ a $451 \mathrm{~nm}$ e ombro a $475 \mathrm{~nm}$. O $R_{F}$ de 0,36, foi compatível com um carotenóide possuindo uma hidroxila e um grupo carbonílico. Foi realizada a reação de redução, resultando em um espectro semelhante ao do $\beta$ caroteno, com $\lambda_{\max }$ a 446 e $473 \mathrm{~nm}$ e ombro a $425 \mathrm{~nm}$. Foram conduzidas as reações de acetilação e metilação, tendo resultado positivo para acetilação e negativo para 
metilação. Duas estruturas eram possíveis, mas considerando a resposta negativa à metilação, a estrutura de 3hidroxi-4'-cetocarotenóide (3-hidroxi- $\beta, \beta$-caroten-4'ona) foi a mais provável.

A sexta fração foi eluída da coluna com $60-70 \%$ de acetona em éter de petróleo, apresentando coloração rósea. Seu espectro, com $\lambda_{\max }$ a 448 e $475 \mathrm{~nm}$ e ombro a $425 \mathrm{~nm}$, foi característico de um carotenóide derivado do $\beta$-caroteno. O Rf de 0,20 foi típico de um diidroxilado. Foram realizadas as reações de metilação e de acetilação, tendo respostas negativa e positiva, respectivamente. Estes resultados identificaram conclusivamente o carotenóide como zeaxantina ( $\beta, \beta$-caroteno-3,3'-diol).

A sétima e última fração foi eluída da coluna com $20 \%$ de água em acetona; sua coloração era laranja clara. Seu espectro foi característico de um cetocarotenóide, $\operatorname{com} \lambda_{\max }$ a $457 \mathrm{~nm}$. Seu $\mathrm{R}_{\mathrm{F}}$ de 0,10 refletiu a presença de duas hidroxilas e um grupo carbonílico. Após a redução, o espectro se transformou em três picos com o $\lambda_{\text {max }}$ a 452 e $481 \mathrm{~nm}$ e um ombro a $423 \mathrm{~nm}$. A resposta à acetilação foi positiva e à metilação negativa. Todas estas características levaram à estrutura 3,3'-diidroxi-4-cetocarotenóide (3,3'diidroxi- $\beta, \beta$-caroten-4-ona).

O cromatograma típico dos carotenóides de amostra saponificada de $S$. pevalekii obtido por CLAE encontra-se na Figura 4. A Tabela I apresenta os $\lambda_{\text {max }}$ obtidos pelo detector de conjunto de diodos dos carotenóides na fase móvel.

Pela ordem de eluição e espectros de absorção obtidos pelo detector de conjunto de diodos, os seguintes carotenóides foram identificados: zeaxantina, 3-hidroxi4 '-ceto-carotenóide, $\beta$-criptoxantina, equininona, trans- $\beta$ caroteno e cis- $\beta$-caroteno. Assim sendo a identificação realizada pelo método CCA foi confirmada pela CLAE. Os isômeros cis e trans do $\beta$-caroteno não separaram na CCA de MgO: Hiflosupercel (1:1). O isômero cis caracterizou-se pelos $\lambda_{\text {max }}$ um pouco menores que os do trans e pela presença do pico cis a $338 \mathrm{~nm}$. O pico 2 apresentou 3 espectros diferentes no início, meio e final do pico, portanto, foi mistura dentro da qual se encontrava um cetocarotenóide (provavelmente 3,3'-diidroxi-4'cetocarotenóide)

Com a amostra não saponificada, os mesmos carotenóides apareceram, ao lado das clorofilas, com exceção do 3-hidroxi-4'-cetocarotenóide, que não foi detectado, pois eluiu junto com a clorofila.

Pelo maior poder de resolução, alguns outros carotenóides foram detectados pela CLAE, mas em quantidades extremamente pequenas, não permitindo a sua identificação.

\section{Carotenóides na Synechocystis pevalekii cultivada sob estresse}

A cianobactéria $S$. pevalekii apresenta-se naturalmente verde, quando cultivada sem fatores limitantes. Quando cultivada sob "stress", no entanto, esta cianobactéria assumiu coloração laranja. Foram identificados apenas um caroteno, um monoidroxicarotenóide e um

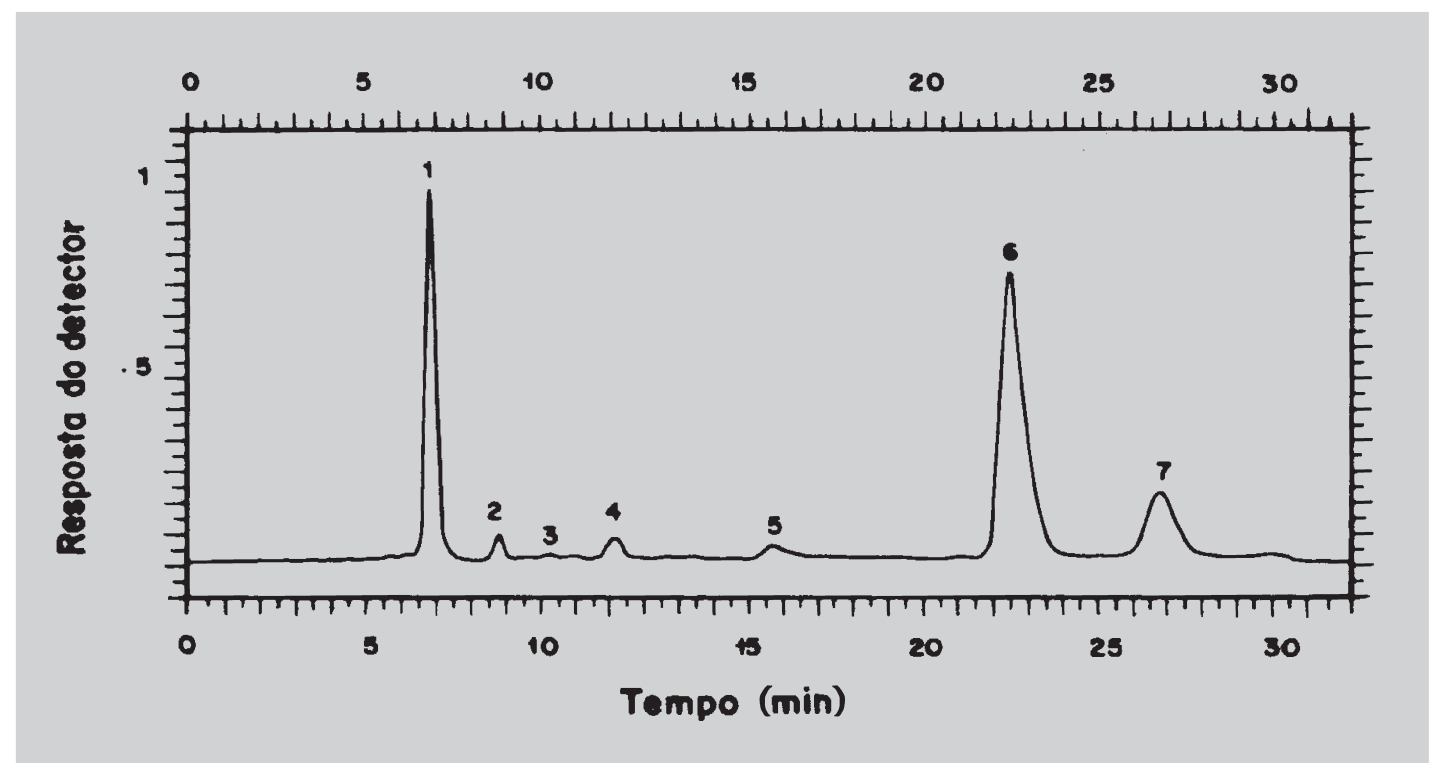

FIGURA 4 - Cromatograma obtido por CLAE dos carotenóides de amostra saponificada de Synechocystis pevalekii. Identificação dos picos: 1. zeaxantina, 2. não identificado, 3. 3-hidroxi-4'-cetocarotenóide, 4. $\beta$-criptoxantina, 5. equininona, 6. trans- $\beta$-caroteno, 7. cis- $\beta$-caroteno. Condições cromatográficas: Coluna $\mathrm{C}_{18}$, Vydac $218 \mathrm{TP} 54,5 \mu \mathrm{m}, 4,6$ mm x $250 \mathrm{~mm}$; fase móvel, metanol:tetraidrofurano (98:2); vazão, $0,8 \mathrm{~mL} / \mathrm{min}$; detector de conjunto de diodos. 
TABELA I - Carotenóides da Synechocystis pevalekii identificados por CLAE com detector de conjunto de diodos

\begin{tabular}{clc}
\hline $\mathbf{N}^{0}$ do pico & $\boldsymbol{\lambda}_{\max }(\mathbf{n m})$ na fase móvel & Identificação \\
\hline 1 & $(426), 449,447$ & zeaxantina \\
2 & $418,445,472$ & Não identificado \\
3 & 461 & 3-hidroxi-4'- \\
& & cetocarotenóide \\
4 & $(426), 449,447$ & $\beta$-criptoxantina \\
5 & 463 & equininona \\
6 & $(426), 449,476$ & trans- $\beta$-caroteno \\
7 & $338,(423), 444,469$ & cis- $\beta$-caroteno \\
\hline
\end{tabular}

*Fase móvel: metanol: tetraidrofurano (98:2).

diidroxicarotenóide na espécie produzida sob estresse. A separação dos carotenóides na coluna de $\mathrm{MgO}:$ Hiflosupercel está apresentada na Figura 5, junto com o comportamento na placa de sílica gel desenvolvida por $5 \%$ de metanol em tolueno.

A primeira, terceira e quarta frações foram identificadas como $\beta$-caroteno, $\beta$-criptoxantina e zeaxantina, respectivamente, pelos mesmos critérios utilizados anteriormente.

A segunda fração foi eluída da coluna com 10-20\% de acetona em éter de petróleo, com uma coloração amarela. Seu espectro mostrou $\lambda_{\max }$ a 376, 400, 426, 448 e 472 nm. O $R_{F}$ foi de um caroteno. Uma recromatografia foi realizada em coluna com diâmetro menor, mas devido à sua baixa quantidade com cor amarela muito clara, não foi possível sua purificação e identificação.

Pela ordem de eluição no cromatograma de CLAE (Figura 6) e espectros de absorção obtidos pelo detector de conjunto de diodos (Tabela II), os seguintes carotenóides

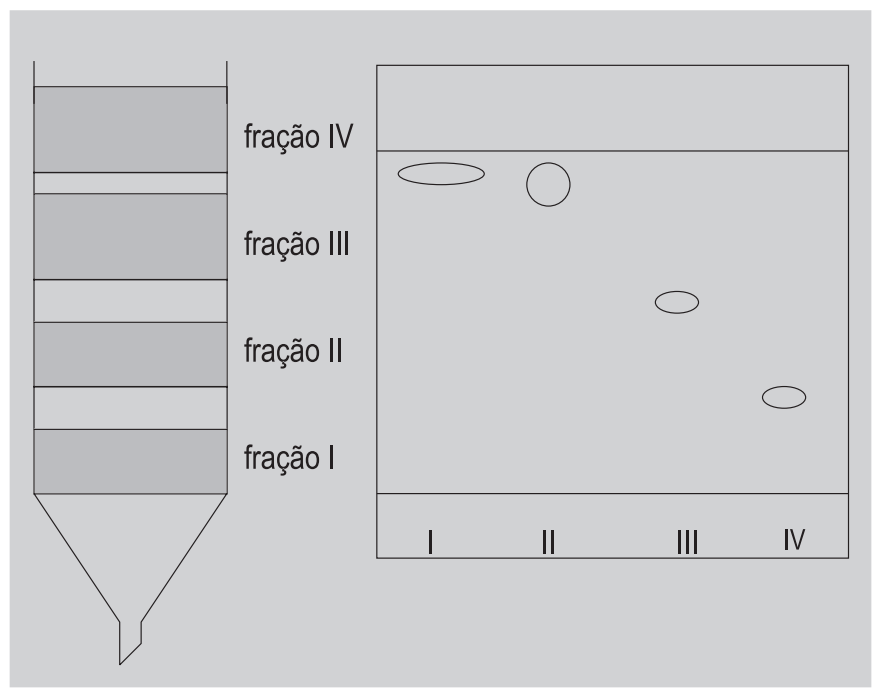

FIGURA 5 - Separação dos carotenóides de S. pevalekii cultivada sob "stress" de nutrientes na coluna de $\mathrm{MgO}:$ Hiflosupercel (1:1) e na placa de sílica-gel desenvolvida por $5 \%$ de metanol em tolueno.

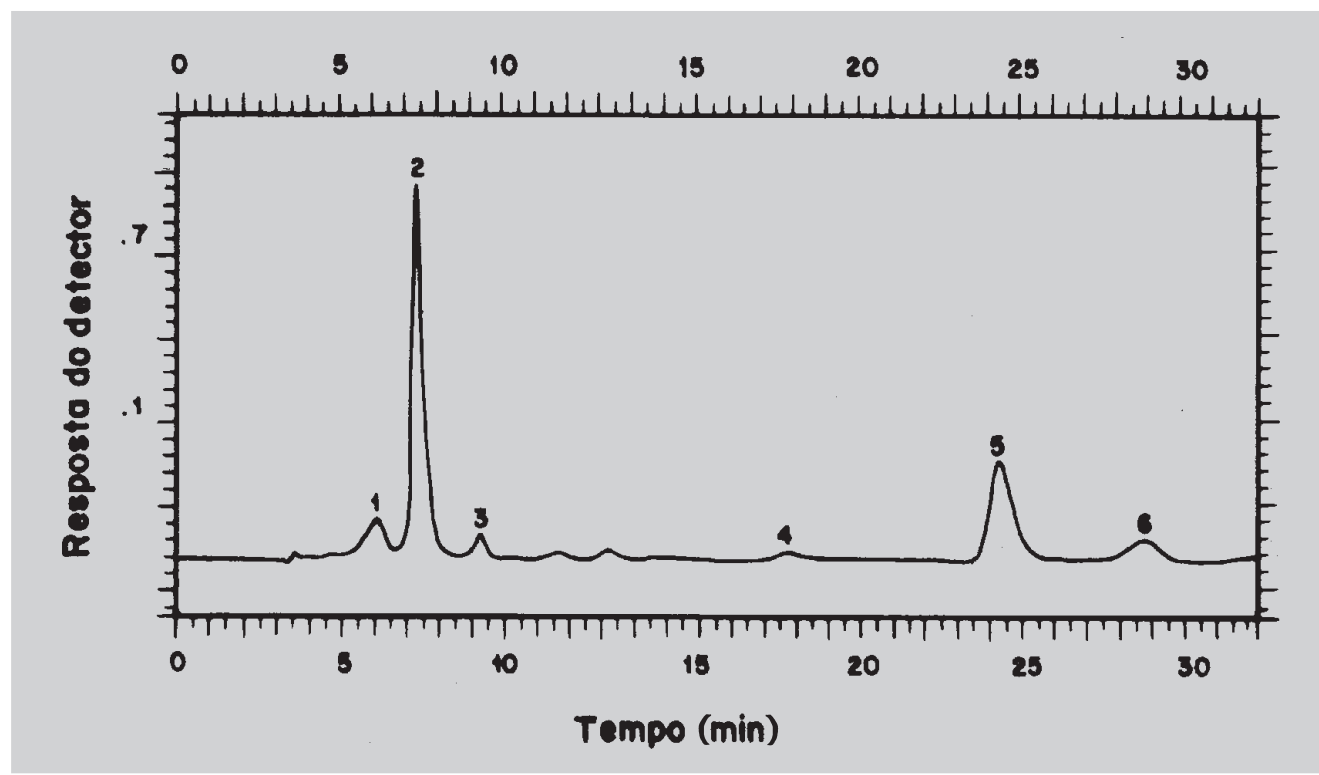

FIGURA 6 - Cromatograma típico dos carotenóides da Synochochystis pevalekii cultivada sob "stress". Identificação dos picos: 1. licofila, 2. zeaxantina, 3. não identificado, 4. mistura, 5. trans- $\beta$-caroteno, 6. cis- $\beta$-caroteno. Condições cromatográficas: Coluna $\mathrm{C}_{18}$, Vydac 218TP54, $5 \mu \mathrm{m}, 4,6 \mathrm{~mm}$ x $250 \mathrm{~mm}$; fase móvel, metanol:tetraidrofurano (98:2); vazão, $0,8 \mathrm{~mL} / \mathrm{min}$; detector de conjunto de diodos. 
foram identificados: licofila, zeaxantina, $\beta$-criptoxantina, trans- $\beta$-caroteno e cis- $\beta$-caroteno. Assim, a identificação de zeaxantina e $\beta$-caroteno pelo CCA foi confirmada pela CLAE.

TABELA II - Carotenóides da S. pevalekii cultivada sob "stress", identificados por CLAE com detector por arranjo de diodos

\begin{tabular}{cll}
\hline $\mathbf{N}^{0}$ do pico & $\boldsymbol{\lambda}_{\max }(\mathbf{n m})$ na fase móvel & Identificação \\
\hline 1 & $446,473,503$ & licofila \\
2 & $426,450,476$ & zeaxantina \\
3 & 450,476 & não identificado \\
4 & $416,440,476$ & mistura \\
5 & $423,450,476$ & trans- $\beta$-caroteno \\
6 & $420,443,473$ & cis- $\beta$-caroteno \\
\hline
\end{tabular}

Fase móvel: metanol: tetraidrofurano (98:2).

A licofila ( $\psi, \psi$-caroteno-16,16'-diol), bem evidenciada na CLAE mas não detectada por CCA, foi identificada pelo espectro de absorção típico de licopeno e tempo de retenção compatível com licopeno hidroxilado. A presença deste carotenóide foi inesperada, uma vez que, em condições normais de crescimento do cianobactéria, não se encontrou este carotenóide.

\section{Composição quantitativa}

As concentrações dos principais carotenóides da cianobactéria $S$. pevalekii encontram-se na Tabela III. Embora não tenha havido diferença em carotenóides totais em $\mu \mathrm{g} / \mathrm{g}$ quantificados na espécie nos dois tratamentos estudados, houve diferença marcante nos teores individuais. O $\beta$-caroteno foi o carotenóide principal da cianobactéria produzida em condições normais, perfazendo $64 \%$ do total, seguido pela $\beta$-criptoxantina. Embora com

TABELA III - Concentrações dos principais carotenóides da cianobactéria Synechocystis pevalekii

\begin{tabular}{lcccc}
\hline Carotenóide & \multicolumn{2}{c}{$\begin{array}{c}\text { Cultivo em } \\
\text { condições normais }\end{array}$} & \multicolumn{2}{c}{$\begin{array}{c}\text { Cultivo sob } \\
\text { "stress" }\end{array}$} \\
& $\boldsymbol{\mu g} / \mathbf{g}$ & $\mathbf{\%}$ & $\boldsymbol{\mu g} / \mathbf{g}$ & $\mathbf{\%}$ \\
\hline$\beta$-caroteno & 307 & 64 & 248 & 52 \\
$\beta$-criptoxantina & 94 & 19 & 13 & 3 \\
3,3'-diidroxi-4" & 51 & 11 & - & - \\
-cetocarotenóide & & & & \\
zeaxantina & 29 & 6 & 220 & 46 \\
\hline TOTAL & 481 & & 481 & \\
\hline
\end{tabular}

menor produção de biomassa, o perfil de carotenóides na cianobactéria produzida sob "stress" de nutrientes foi muito interessante. Houve um decréscimo substancial de $\beta$-caroteno (de 307 para $248 \mu \mathrm{g} / \mathrm{g}$ ) e aumento considerável de zeaxantina (de 29 para $220 \mu \mathrm{g} / \mathrm{g}$ ), sendo estes dois os carotenóides majoritários, contribuindo com 52 e $46 \%$ do total, respectivamente. Houve também diminuição da $\beta$-criptoxantina (de 94 para $13 \mu \mathrm{g} / \mathrm{g}$ ).

$\mathrm{O} \beta$-caroteno é a pró-vitamina $\mathrm{A}$ mais potente, à qual é atribuída $100 \%$ de atividade. Embora não seja uma pró-vitamina A, a zeaxantina, é o carotenóide, junto com a luteína, especificamente implicado na diminuição do risco de catarata e degeneração macular, principal causa de cegueira em idosos. Os dois, portanto, desempenham papéis importantes na saúde humana. No entanto, enquanto já existem várias fontes comerciais de $\beta$-caroteno, a zeaxantina não é facilmente encontrada, especialmente em quantidades compatíveis com a utilização comercial.

\section{CONCLUSÃO}

A cianobactéria S. pevalekii tem potencial comercial. Em condições normais, esta espécie apresenta, como carotenóide principal, o $\beta$-caroteno. Na cianobactéria produzida sob stress há decréscimo do $\beta$-caroteno e aumento significativo da zeaxantina. Portanto, as condições de produção da espécie podem ser estabelecidas de tal forma que a biossíntese de carotenóides importantes a saúde humana e de difícil obtenção seja favorecida.

\section{ABSTRACT}

\section{Carotenoids of the cyanobacterium Synechocystis pevalekii produced under normal conditions and under nutrient limitation}

The use of microalgae and cyanobacteria as sources of nutrients and bioactive substances for food and dietary supplements has attracted a lot of interest in recent years. Through open column chromatography-visible absorption spectrophotometry, high performance liquid chromatography with a photodiode array detector, thin layer chromatography and functional group chemical reactions, trans- and cis- $\beta$-carotene, echinenone, $\beta$-cryptoxanthin, 3 hydroxy-4'-ketocarotenoid, zeaxanthin and 3,3dihydroxy-4'-ketocarotenoid were identified in the cyanobacterium Synechocystis pevalekii. The cianobacterium was green because of the presence of chlorophylls. When cultivated under stress $(80 \%$ reduction of nutrient content of the original Conway medium) the chlorophylls disappeared and the cyano- 
bacterium assumed an orange color. $\beta$-Carotene decreased from 307 to $248 \mu \mathrm{g} / \mathrm{g}$ and $\beta$-cryptoxanthin from 94 to $13 \mu \mathrm{g} / \mathrm{g}$. On the other hand, zeaxanthin increased from 29 to $220 \mu \mathrm{g} / \mathrm{g}$. Thus, S. pevalekii appears to have commercial potential as source of zeaxanthin, which is implicated in the reduction of the risk of macular degeneration and cataract, together with lutein. The results also showed that conditions for the production of the cyanobacterium can be established so that the biosynthesis of carotenoids important to human health, but difficult to obtain, can be favored. There are already several commercial sources of $\beta$-carotene, but sources of zeaxanthin are rare.

UNITERMS: Cyanobacterium. Synechocystis pevalekii. Carotenoids. Zeaxanthin.

\section{AGRADECIMENTOS}

Ao CNPq, pela bolsa de iniciação científica concedida ao primeiro autor e ao MCT-CNPq-FINEP, pelos recursos financeiros através do Projeto PRONEX no. 4196091500/CNPq no. 662307/1996-8.

\section{REFERÊNCIAS BIBLIOGRÁFICAS}

BEN-AMOTZ, A.; KATZ, A.; AVRON, M. Accumulation of $\beta$-carotene in halotolerant algae: Purification and characterization of $\beta$-caroteno-rich globules from Dunaliella bardawii.J. Phycol., Malden, v. 8, p. 529-537, 1982.

BOROWITZKA, M.A. Products from microalgae. Infofish Inter., Kuala Lumpur, v. 5, p. 21-26, 1993.

BOROWITZKA, M.A. Vitamins and fine chemicals from microalga. In: BOROWITZKA, M.A., BOROWITZKA, L.Y., eds. Microalgal biotechnology, Cambridge: Cambridge University Press, 1988. p. 153161.

BRITTON, G. UV/visible spectroscopy. In: BRITTON, G.; LIAAEN-JENSEN, S.; PFANDER, H., eds. Carotenoids: Spectroscopy. Basel: Birkhäuser Verlag, 1995. v. 1B, p. 13-63.
DAVIES, B.H. Carotenoids. In: GOODWIN, T.W., ed. Chemistry and biochemistry of plant pigments. London: Academic Press, 1976. v. 2, p. 38-165.

GOUVEIA, L.; REIS, A.; VELOSO, V.; EMPIS, J.A. Microalgal biomass as a sustainable alternative raw material. Agro-Food-Industry Hi-Tech., Milano, v. 3 , p. 29-34, 1996.

KAY, R.A. Microalgae as food and supplement. Crit. Rev. Food Sci. Nutr., Boca Raton, v. 30, p. 555-573, 1991.

KRINSKY, N.I. Actions of carotenoids in biological systems. Annu. Rev. Nutr., Palo Alto, v. 13, p. 561-587, 1993.

MAYNE, S.T. $\beta$-Carotene, carotenoids and cancer prevention in humans. FASEB J., Bethesda, v. 10, p. 690-701, 1996.

OLSON, J.A. Carotenoids. In: SHILS, M.E.; OLSON, J.A.; SHIKE, M.; ROSS, A.C., eds. Modern nutrition in health and disease. 9. ed. Baltimore: Williams \& Wilkins, 1999. p. 525-541.

OROSA, M.; VALERO, J.F.; HERRERO, C.; ABALDE, J. Comparison of the accumulation of astaxanthin in Haematococcus pluvialis and other green microalgae under N-starvation and high light conditions. Biotechnol. Lett., Dordrecht, v. 23, p. 1079-1085, 2001.

RISE, M.; COHEN, E.; VISHKAUTSAN, M.; COJOCARU, M.; GOTTLIEB, H.E.; ARAD, S.M. Accumulation of secondary carotenoids in Chlorella zofingiensis. J. Plant Physiol., Stuttgart, v. 144, p. $287-$ 292, 1994.

RODRIGUEZ-AMAYA, D.B. A guide to carotenoid analysis in foods. Washington: ILSI Press, 1999. 64 p.

WALNE, P.R. Experiments in the large scale culture of the larvae of Ostrea edulis. Fishery Invest., London, v. 25, p. $1-53,1966$.

Recebido para publicação em 31 de março de 2003. 